\title{
Leptomeningeal Carcinomatosis with Delayed Ocular Manifestations
}

\author{
Craig Czyz Kyle Blair Reece Bergstrom \\ Division of Ophthalmology, Section Oculofacial Plastic and Reconstructive Surgery, Ohio \\ University/OhioHealth Doctors Hospital, Columbus, OH, USA
}

\section{Keywords}

Leptomeningeal carcinomatosis · Ophthalmology · Cranial neuropathy · Optic neuropathy .

Vision loss · Magnetic resonance imaging

\begin{abstract}
The most common presenting symptoms of leptomeningeal carcinomatosis (LC) are ocular manifestations with vision loss. There are multiple reports of undiagnosed LC causing sudden, complete, monocular vision loss as the presenting symptom; however, sudden bilateral vision loss 6 months following diagnosis has not been described. Any new ocular involvement or worsening of previous ocular symptoms warrants reimaging and prompt ophthalmology consultation, as this likely indicates disease progression. This report details a unique case of LC where a previously diagnosed patient developed sudden, complete, bilateral vision loss and multiple cranial nerve palsies with progression of LC on imaging.

\section{Introduction}

Leptomeningeal carcinomatosis (LC) is a severe and rare complication of both solid and blood malignancies. The disease manifests from the dissemination of malignant cells that seed the cerebrospinal fluid, inner two layers of meninges, arachnoid and pia mater, and/or leptomeninges [1]. The incidence of $\mathrm{LC}$ is about $5 \%$ of known malignancies and disease occurrence is associated with several clinical risk factors including: age less than 60, hypoalbuminemia, involvement of the testis, breast, or bone marrow [2]. Patients can develop a myriad of neurological symptoms due to central nervous system involvement. Central nervous system symptoms may include seizures, encephalopathic states, hemiparesis, aphasia, or cranial nerve palsies. However, commonly the presenting clinical feature of LC is ocular 
involvement. In a systematic review, $83 \%$ of patients presented with ocular symptoms [3]. Ocular symptoms include but are not limited to diplopia, vision loss, ptosis, papilledema, anisocoria, exophthalmos, orbital pain, and hemianopsia. Vision loss was the most common presenting ocular symptom and occurred in $50 \%$ of patients [3].

Case Presentation

A 33-year-old Caucasian female with a past medical history of triple negative stage IV breast cancer with metastasis to brain with known leptomeningeal disease for 6 months, bilateral mastectomy followed by complete chest wall radiation, and systemic chemotherapy reported sudden painless vison loss. The patient initially presented to the emergency department with breakthrough seizure activity with subsequent hospitalization for evaluation. On day 2 of admission, neurology assessed the patient and a left homonymous hemianopsia was noted. The following day, the patient developed left eyelid ptosis and anisocoria. A brain magnetic resonance imaging (MRI) scan was ordered by neurology and showed no evidence of hydrocephalus, leptomeningeal metastasis unchanged, and overall stable periventricular/subcortical white matter post treatment. On day 4, the patient lost vision in both eyes and was encephalopathic. Ophthalmology was then consulted.

Ophthalmology examination revealed visual acuity was no light perception bilaterally. Pupils were minimally reactive, with the left larger than the right. The left upper eyelid had severe ptosis, covering the pupil. On exam, there was no evidence of disc edema and the fundus appeared normal. An MRI of the orbits with and without contrast showed a small amount of fluid and enhancement surrounding the optic nerves. In addition, there was apparent enhancement involving the bilateral 7 th and 8th nerve complexes.

\section{Discussion}

There are multiple case reports in the literature of undiagnosed LC causing sudden, complete binocular vision loss as the presenting symptom; however, sudden bilateral vison loss 7 months following diagnosis has not been described as indicative of active progression $[2,4,5]$. A case series of two patients with blindness as an initial manifestation of LC from breast cancer postulates that the vision loss could be due to a combination of chronic papilledema, cuffing of the tumor around the optic nerve, as well as direct infiltration [6]. Direct infiltration of the optic nerve was also proposed as a mechanism for sudden blindness in a patient with LC due to rectal cancer [7]. This patient had bilateral enhancement of the optic nerve sheaths on MRI with an afferent pupillary defects; however, no optic nerve edema was seen, supporting direct infiltration and/or nerve cuffing as the cause of optic nerve damage. LC also has been known to cause simultaneous 7 th and 8th nerve palsies due to infiltration of the internal auditory meatus, as was the case in this patient. Involvement of multiple of these cranial nerves predicates CSF involvement [8].

The sudden progression of previously diagnosed LC to complete vision loss in a patient is atypical. This may be in part due to advancing chemotherapy, or due to the relatively short lifespan of patients once diagnosed, with a median survival of 4 months [9]. This patient had been diagnosed with LC approximately 7 months prior to having this manifestation. Patients with ocular manifestations often have other contemporaneous neurologic signs, as was the case with this patient, but can present solely with ocular signs as well [10].

In consideration of this case, ophthalmology should be involved with the patient's care if there are any ocular symptoms. These ocular signs include diplopia, ptosis, papilledema, anisocoria, exophthalmos, orbital pain, scotomas, hemianopsia, and nystagmus. Multiple other cranial nerves can be involved as well. Visualization of the fundus and optic nerve can

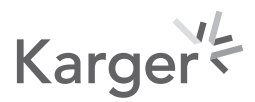


help differentiate whether a patient has vision loss associated with another manifestation of metastatic disease, such as cancer-associated retinopathy or paraneoplastic optic neuropathy. Any new ocular signs or a worsening of previous ocular signs warrants reimaging in patients with a diagnosis of LC, as this likely indicates progression. As shown in this case, progression can happen suddenly and affect both optic nerves, so an urgent ophthalmology referral is recommended.

\section{Statement of Ethics}

The subject has given her written informed consent to publish this case (including publication of images). No IRB approval was needed for this case report. This study complied with all aspects of the World Medical Association Declaration of Helsinki.

\section{Conflict of Interest Statement}

The authors declare no conflicts of interest.

\section{Funding Sources}

No funding was received for this work.

\section{Author Contributions}

Craig Czyz: conceptualization, methodology, reviewing, editing, supervision. Kyle Blair: conceptualization, methodology, writing, editing, investigation, primary contact. Reece Bergstrom: conceptualization, methodology, writing, editing, investigation.

\section{References}

1 Bussat A, Langner-Lemercier S, Salmon A, Mouriaux F. Paraneoplastic syndromes in ophthalmology. J Fr Ophtalmol. 2018 May;41(5): e181-5.

2 Huang YC, Wang CJ, Jou JR. Blindness due to leptomeningeal carcinomatosis as an initial manifestation of recurrent acute lymphoblastic leukemia. Taiwan J Ophthalmol. 2019 Dec 13;9(4):288-91.

3 Lanfranconi S, Basilico P, Trezzi I, Borellini L, Franco G, Civelli V, et al. Optic neuritis as isolated manifestation of leptomeningeal carcinomatosis: a case report and systematic review of ocular manifestations of neoplastic meningitis. Neurol Res Int. 2013;2013:892523.

4 Akritidou F, Karafyloglou M, Karamanis D. A 56-year-old man with acute vision loss. Digit J Ophthalmol. 2016 Aug 18;22(3):68-71.

5 Sabater AL, Sadaba LM, de Nova E. Ocular symptoms secondary to meningeal carcinomatosis in a patient with lung adenocarcinoma: a case report. BMC Ophthalmol. 2012;12:65.

6 Cantillo R, Jain J, Singhakowinta A, Vaitkevicius VK. Blindness as initial manifestation of meningeal carcinomatosis in breast cancer. Cancer. 1979;44(2):755-7.

7 McFadzean R, Brosnahan D, Doyle D, Going J, Hadley D, Lee W. A diagnostic quartet in leptomeningeal infiltration of the optic nerve sheath. J Neuroophthalmol. 1994 Sep;14(3):175-82.

8 Imamura S, Nozawa I, Imamura M, Murakami Y. Clinicopathologic study of leptomeningeal carcinomatosis involving the temporal bone. Ann Otol Rhinol Laryngol. 1997;106(8):674-9.

9 Franzoi MA, Hortobagyi GN. Leptomeningeal carcinomatosis in patients with breast cancer. Crit Rev Oncol Hematol. 2019 Mar;135:85-94.

10 Mayer RR, Frankfort BJ, Strickland BA, Debnam JM, McCutcheon IE, Groves MD, et al. Leptomeningeal metastases presenting exclusively with ocular disturbance in 34 patients: A tertiary care cancer hospital experience. J Clin Neurosci. 2017;39:151-4. 